\title{
Urgences
}

\section{L'intimité Proust}

\section{Marcel Proust, À la recherche du temps perdu, Paris, Gallimard, coll. " Bibliothèque de la pléiade ", 1954, tome 1, 2 et 3.}

\section{Hughes Corriveau}

Numéro 23, avril 1989

Lisière du livre

URI : https://id.erudit.org/iderudit/025525ar

DOI : https://doi.org/10.7202/025525ar

Aller au sommaire du numéro

\section{Éditeur(s)}

Urgences

ISSN

0226-9554 (imprimé)

1927-3924 (numérique)

Découvrir la revue

Citer ce compte rendu

Corriveau, H. (1989). Compte rendu de [L'intimité Proust / Marcel Proust, À la recherche du temps perdu, Paris, Gallimard, coll. " Bibliothèque de la pléiade ", 1954, tome 1, 2 et 3.] Urgences, (23), 143-147. https://doi.org/10.7202/025525ar d'utilisation que vous pouvez consulter en ligne.

https://apropos.erudit.org/fr/usagers/politique-dutilisation/ 


\section{deliveline}

Marcel Proust, À la recherche du temps per$d u$, coll. «Bibliothèque de la pléiade", Paris, Gallimard, 1954, tome 1, 1003 p.; tome 2, 1224 p.; tome 3, 1324 p.

George D. Painter, Marcel Proust, tome 1, 1871-1903: Les années de jeunesse et tome 2, 1904-1922: Les années de maturité, Paris, Mercure de France, 1966, 466 p. et 517 p.

\section{Philippe Boyer, Le petit} pan de mur jaune. Sur Proust, coll. «Fiction \& Cie", Paris, Seuil, 1987, 252 p.

\section{Vincent Descombes, Proust. Philosophe du roman, coll. «Critique», Paris, Minuit, 1987, 338 p.}

\section{L'INTIMITÉ PROUST}

«Longtemps je me suis" fermé à Marcel Proust. "Parfois, à peine" avais-je le livre entre les mains, «mes yeux se fermaient si vite que je n'avais pas le temps de me dire: "Je m'endors". Et, une demi-heure après, la pensée qu'il était temps de chercher» une explication à ma résistance «m'éveillait; je voulais poser le volume que je croyais avoir entre les mains et souffler ma lumière; je n'avais pas cessé en dormant de faire des réflexions sur ce que je venais de lire, mais ces réflexions avaient pris un tour un peu particulier; il me semblait que j'étais moi-même exclu de ce dont parlait l'ouvrage». Que de temps je mettrai avant d'en saisir le premier mot, la première ligne, la première page. L'aventure a commencé je devais avoir quinze ans. Mon frère et moi, nous nous faisions un devoir de nous acheter au moins un livre de poche chacun chaque semaine afin de pouvoir profiter ainsi d'une lecture double, complice, heureuse. Je ne sais quel diable me poussera alors à m'acheter l'horrible volume de la collection de poche intitulé Un amour de Swann. D'abord je trouvais le titre très laid et rien ne m'attirait dans la présentation matérielle de ce texte. Je commençai donc alors une longue série, mais très longue série de lectures, de débuts de lecture, de tentatives de lecture, et de relectures. Le remords s'était insidieusement emparé de moi, la culpabilité, le sentiment d'être un imbécile, un ignorant, un Wisigoth, quelque spécimen intellectuel obtus, fermé aux usages du beau langage, de la belle phrase, du beau style. 
Quand je commençais à lire: "Pour faire partie du "petit noyau", du "petit groupe", du "petit clan"”, je me mettais à frissonner tant la pensée d'en justement faire partie me rebutait comme m'aurait rebuté l'idée de me joindre à un club de hockey, ou à quelques groupes de "bums" du quartier. Non, décidément, je n'allais pas faire partie des adorateurs, et je me faisais fort de ne jamais, mais au grand jamais, lire Proust. II m'étouffait dans son style, il me faisait aussi peur qu'il me fascinait. J'étais déjà sous le charme et comme il se doit, j'avais l'adolescence difficile et orgueilleuse, je n'allais donc pas accepter la séduction. Je ne sais par quelle contagion, mon frère aussi n'y prit pas plus de goût que moi, et nous évitâmes alors l'infernale série des tomes qu'on publiait de temps à autre, à seule fin, eût-on dit, de me narguer, de me montrer l'infranchissable abîme qui me séparait du savoir. Le meilleur ami que j'avais alors (et que j'ai encore malgré tout, après trente-cinq ans de connaissance!), cet ami donc me fitl'affront de s'acheter La recherche en entier, et dans la collection "Blanche" encore (combien de tomes là-dedans?). Encore s'il s'était contenté de l'acheter, d'en décorer orgueilleusement un rayon ou deux de sa bibliothèque montée sur briques, mais il a fallu qu'il la lise, au complet, avec délectation, avec jouissance, avec compétence! J'ai poussé l'intransigeance jusqu'à me moquer, jusqu'à lui interdire de m'en parier jamais, sous le fallacieux prétexte que cela ne pouvait m'intéresser. J'avais ainsi des libertés assez radicales avec ce qui devait ou ne devait pas me préoccuper. Quand ce fut le tour de Paul Chanel Malenfant d'y prendre aussi un plaisir certain, je me suis senti cerné de toutes parts. II m'aurait fallu des alliés, des appuis, de ces préhistoriques congénères qui n'auraient pas lu, eux, le Livre, l'indispensable. N'avais-je pas reçu un présage de la part de Laurent Mailhot alors que je passais un examen oral de lecture, justement au sujet du premier livre de La recherche, livre choisi parmi plus de cent vingt titres que nous devions lire cette année-là, et le seul sans doute que je n'avais pas lu. Était-ce possible qu'alors que j'avais lu cent dix-neuf titres différents, étaitce possible que Laurent Mailhot choisisse justement celui-là, le damné, le misérable Proust? Que pouvais-je répondre, sinon avouer l'infamie, avouer mon ignorance, avouer que je n'avais pas pu, que «je n'aimais pas Proust"? II aurait fallu voir la tête des examinateurs, regarder leurs fronts glabres, leur savantissime stupéfaction.

Laurent Mailhot de me promettre alors que «j'y viendrais, qu'un jour je finirais bien par aimer, lire, me rendre jusqu'au bout". Serais-je capable de traduire ici les frissons angoissés qui me parcoururent alors le dos devant ce qui me paraissait être une invraisemblable méchanceté, car, à mes yeux, on ne pouvait pas souhaiter pire à quelqu'un? Et voilà! II aura eu raison! J'y suis venu, et j'ai réussi, je ne sais pas encore comment, mais à aimer, à aimer comme rarement on aime des livres, avec une passion tranquille, intime, une conviction si profonde de l'importance de l'oeuvre qu'il nous semble bien inutile parfois d'en parler plus, tant on aimerait laisser ce livre nous habiter, nous envahir tout doucement, intimement dans le plaisir satisfait. J'ai lu Proust, et plus encore, chose incroyable, j'ai aimé lire Proust. Dirais-je aussi que c'est le livre qui m'a sans doute fait le plus de bien dans ma vie?

Comment cela est-il arrivé? Tout à fait par hasard, dans un moment de grande déprime, quand tout allait mal pour je ne sais plus quelle raison ( $\mathrm{je}$ m'invente un si grand nombre de raisons d'être mal avec moi-même que j'en viens à ne plus me rappeler telle ou telle), dans un de ces moments où le goût de tendre la main vers un livre devient tout à fait irrésistible. J'ai pris le premier tome de "La Pléiade" parce que c'était gros, parce que je savais que ça me tomberait des mains comme tout le reste, comme à 
chaque fois que je l'avais tenu. Et voilà. J'ai lu la première phrase qui a une ligne, puis la seconde qui en a douze, et j'ai compris alors ce qui depuis tant d'années m'avait empêché d'entrer là dans le «clan»: la phrase Proust! C'est elle qui m'avait fermé cet univers qui pourtant ne tient tout entier qu'à elle, qu'à ce qu'elle recèle de subtilité, de méandres. J'avais été pendant des années fasciné par un style, obnubilé par lui, jusqu'à ne jamais saisir qu'il se racontait là une histoire, une réelle histoire, qu'il n'y avait pas que de la phrase, que de la ponctuation, que de la compétence à faire rougir tout ignorant. Ce soir-là, j'ai dépassé la complexité si belle, si réelle, de la phrase et j'ai consenti à cet acte d'humilité si terrible qui consiste à accepter de se faire raconter une histoire. Je me suis fait raconter une histoire d'enfant insomniaque et jaloux, d'un enfant amoureux et curieux, insatiable, raffiné, jouisseur, pour tout dire un enfant qui allait tranquillement m'ouvrir les phrases qui jusque-là m'avaient refusé. Et si lire Proust peut alors commencer par un événement de séduction, j'avouerai que le charme alors aura opéré. Cela aura pris exactement vingt ans entre ma première rencontre avec l'oeuvre et mon orgueilleux refus d'alors. L'instant magique m'aura permis de lire ces phrases où cette nuit terrible dévoile les secrets des adultes, en bas dans le jardin, alors qu'ils s'y tenaient sans moi depuis tant d'années.

Comment répondre après tant d'autres à la question de savoir ce qu'il y a tant là qui fasse époque, fasse urgence au point d'imposer ce texte comme un des plus grands du siècle? Comment y répondre sinon en parlant pour soi, exclusivement, du côté de l'émotion, de la prise de la parole, de l'emprise subjective qui s'opère ici au texte donné comme définitif? Lire Proust, c'est faire accord de soi et d'un texte cui parle de l'être, d'une langue qui jouit de dire la pensée fulgurante, fuyante, fragile des personnages mis ici en prises, donnés. Comment ne pas reconnaître cette façon Proust d'être indiscret, cette voyance étrange dans ce qu'il offre de l'esprit, son travail, son inquiétude, son abandon? Faut-il redire la pénétrante enquête qui s'y mène au lieu même du sensible, de ce qui fait une société policée, cultivée? Proust, ce pourrait être l'art du bonheur, de la surprise, de l'étonnement devant le monde. Proust, ce pourrait être cette évocation du moment idéal qui surgit dans chaque partie du corps comme un lieu précis de mémoire, car chez Proust, la pensée est plus que la pensée, elle est sensation vive, force émotionnelle. Une pensée qui donc redonne le monde à qui sait s'attarder au palpable, au soyeux des gestes, aux mystérieuses incidences de l'art, de l'oeil, des sens. Proust, ce serait avant tout une manière d'être au monde dans l'échancrure même des matières, oeil ouvert sur le possible, à jamais concerné par la vie, toute vie, tout mot, toute immédiateté de l'amour. Car on aime chez Proust comme ça s'écrit, cette vie témoigne du bonheur d'exister dans l'inquiétude de soi comme des autres, l'inquiétude devenant un mode d'existence privilégié, d'ouverture au monde, de vives attentes des gestes, des autres, des êtres. Proust serait cela, cette écriture toujours renouvelée qui dit et redit l'existence à travers la tension permanente qu'il faut assouvir pour parvenir à une relative tranquillité. Proust dit l'existence des autres en dehors de toute solitude. II rend la vie des autres essentielle en ce qu'elle est toujours susceptible de donner l'amour essentiel au narrateur. Et ce narrateur, n'estil autre que ce désir devenu lecteur, que cette tension qui nous porte à ne pas finir l'oeuvre, à la trouver toujours sur son chemin avec son poids de mots, sa fatalité de mots, son envergure imprenable, son existence démesurée, comme le monde lui-même qui nous convie chaque jour à vivre? Proust, ne serait-il en fin de compte que ce jeu de regards qui rend la vie toujours à elle-même plus essentielle? Ne serait-il pas cette oeuvre 
même qui la dit, la recrée, la jauge au poids même des mots, dans la fabrique même des lieux? Proust n'est-il pas la toponymie essentielle dont chacun rêve, n'est-il pas celui qui nomme les lieux de passages, de toute une vie de passages comme on aimerait bien faire soi-même pour chacun des lieux de notre existence, pour chacun des lieux qui nous envahissent à chaque heure, à chaque jour? Proust, ne serait-ce pas cette écriture qui n'en finit pas de décrire du vêtement, du corps, du salon, du paysage, de l'oeuvre d'art, de cette sorte de prière qui se tient tout entière dans un mot d'amour? Proust, ne serait-ce pas cette manière de faire de la littérature le champ entier de la réserve de soi, le non-perdu, ce qui se sauve à la fin de tout ce qui s'écoule, se perd? Proust, n'aurait-il pas trouvé dans l'excès d'histoire, dans la fragilité même de ce qu'il dit, le mot même de l'existence, cette sorte de morceau vivant qui fait qu'une vie se vit de rien, de tous petits riens, d'un passage à peine entamé sur le pan entier, et peut-être jaune, d'un siècle?

Lire la vie de Proust dans le Painter n'ajoute rien à l'oeuvre mais offre à qui s'est pris d'amour pour lui le moment de grâce de croire un instant le rejoindre dans le réel. Illusion toujours nécessaire des biographies que cette façon de nous faire croire un instant à l'ordre chronologique, à sa vive précarité. Lire la vie de Proust, c'est en quelque sorte consentir à se désenchanter, à faire table rase pour un peu de temps de la grandeur de l'oeuvre elle-même. Le magique disparaît, pour ne laisser place qu'à l'existence faussement donnée pour réelle, faussement racontée comme s'il était possible de circonscrire le passé Proust autrement que par la mémoire involontaire, ce qui nous est, à nous, parfaitement impossible puisque nous n'avons pas sous le pied ce souvenir inconfortable des pavés de Venise. $Y$ a-t-il auteur moins en accord avec l'idée d'une biographie que Proust? En lisant le Painter, je me disais que je faisais en quelque sorte une trahison, comme s'il ne me suffisait pas d'avoir lu le livre, comme s'il fallait me détacher de l'illusion créée par La recherche pour parvenir enfin à donner une mesure plus humaine au personnage. Mais est-il vraiment possible de retracer son plaisir aussi froidement que dans la mise ensemble d'une chronologie froide et achevée? Rien n'y a fait. Je préfère penser que Combray existe quelque part en moi plus certainement que Cabourg, plus justement dans l'illusion dramatique de toute oeuvre de fiction.

Mieux vaut alors lire attentivement le Descombes. Y chercher une intelligence remarquable de l'oeuvre. "ll y a d'abord l'image d'un livre intérieur qui a été mal traduit au cours de la vie, et que l'écrivain doit traduire à nouveau", dit-il quelque part. $\mathrm{Ne}$ serait-ce pas dans ce malentendu que nous entretenons avec notre propre vie, notre propre existence, que se situerait cette pulsion insensée qui nous pousse, écrivains, à nous décharger de cet incompris fondamental dans cette fiction à jamais recommencée de nos livres, du cumul que nous en faisons? Proust n'aurait-il pas écrit cette méprise qui le tenait prisonnier du temps présent alors que tout son désir tenait au pouvoir absolu d'un surgissement inévitable du passé dans le présent, de la fulgurance du souvenir actualisé dans son involontaire résurgence? Avoir lu Proust, c'est aussi consentir à un itinéraire qui nous conduit parfois chez ses exégètes. Une faim de ne jamais en finir nous fait considérer cette oeuvre comme le mur même sur lequel on ne voudrait ne jamais achever sa contemplation, son bonheur. Avoir lu Proust, c'est passer de Descombes à Philippe Boyer, pour savoir un peu plus ce que d'autres y ont vu, y ont compris, c'est ne pas laisser le temps passer sur notre propre lecture, de telle sorte qu'on puisse avoir l'illusion de la redonner comme présente. Avoir lu Proust, c'est aussi ne pas s'en sortir tout à fait comme je le fais maintenant en écri- 
vant un recueil de nouvelles à partir de citations de Proust, pour que queique part il ait part une fois à mon écriture, en toute prétention, en toute perversion. Avoir lu Proust, c'est consentir à ce que ce livre-là, ce Proust-là, ne passe pas, ne s'achève pas.

Hugues Corriveau 